\title{
Capturing Four Typical Eye Movement Signals Hidden in Electroencephalograph
}

\author{
Liang-zhi GAN ${ }^{1}$, Rong-gang ZHAO $^{2}$, Hai-kuan LIU*1 \\ ${ }^{1}$ School of Electrical Engineering and Automation, Jiangsu Normal University, Xuzhou, China \\ ${ }^{2}$ Defense Department, Jiangsu Normal University, Xuzhou, China \\ ${ }^{*}$ Corresponding author
}

\begin{abstract}
Keywords: Principal Angles; Kernel Method; Electroencephalograph
Abstract. We proposed a process to find and recognize four typical eye movement signals hidden in EEGs. Eye movement signals contained in EEG are usually looked as artefacts by doctors. But they can be useful if the movements are intentionally launched for special purpose. Identifying typical eye movements from a flow of EEG signals can facilitate communications. We introduce the way to identify four typical eye movement signals by analyzing the electrooculography (EOG) signals with kernel principal angles. Supposing one person tries to give signal by moving eyes, it is convenient for him or her to move the eyes left, right, up or down. We sampled many typical EOG signals of these special conditions. Kernel principal angles are often used to measure the similarity of two data sets. With the help of kernel principal angles, we tried to capture the eye movement signals hidden in EEG signals and recognize them. The final experiments show that overwhelming majority of the cosines in the same classes are over 0.95 , and cosines over different classes are less than 0.80 . This means that kernel principal angles can be effective to capture and identify typical eye movement signals.
\end{abstract}

\section{Introduction}

Principal angles propose a method to measure the similarity of two data sets [1]. But this method is seldom used, or, it is not popular until the appearance of kernel skill [2]. It is very common to inspect the relation between two random variables $\mathrm{X}$ and $\mathrm{Y}$. In most cases, we can not get their model or information. So we can estimate the relation by data sets $A=\left\{x_{1} ; L ; x_{m}\right\}$ and $B=\left\{y_{1} ; L ; y_{n}\right\}$ (samples from the random variables $\mathrm{X}$ and $\mathrm{Y}$ ). These two sets can span two spaces which are denoted as $\mathrm{U}_{\mathrm{A}}=\operatorname{span}\left\{x_{1} ; \mathrm{L} ; x_{\mathrm{m}}\right\}=\left\{u: u=\sum_{i=1}^{m} \alpha_{i} x_{i}, \alpha_{i} \in \mathrm{R}\right\} \quad$ and $\quad \mathrm{U}_{\mathrm{B}}=\operatorname{span}\left\{y_{1} ; \mathrm{L} ; y_{\mathrm{n}}\right\}=\left\{v: v=\sum_{i=1}^{n} \beta_{i} y_{i}, \beta_{i} \in \mathrm{R}\right\}$. The principal angles between the two subspaces are described by the cosines as [1]:

$$
\cos (\theta)=\max _{\mathrm{u} \in \mathrm{U}_{\mathrm{A}}, v \in \mathrm{U}_{\mathrm{B}}} \mathrm{u}^{\mathrm{T}} \mathrm{v},
$$

subject to

$$
\mathrm{u}^{\mathrm{T}} \mathrm{u}=\mathrm{v}^{\mathrm{T}} \mathrm{v}=1 \text {. }
$$

It is easy to understand that principal angles describe the minimum angle between two spaces. The principal angles have two critical defects. The first is restricted to small data sets because as the increase of the elements in sets $A$ and $B$, it will surely be true that $U_{A} I U_{B} \neq \Phi$. This means the two spaces $U_{A}$ and $U_{B}$ overlap, $\cos (\theta)=1$. On this case, principal angles can not measure the similarity of data sets. The second defect is that it can only measure linear relations because $U_{A}$ and $U_{B}$ are linear combination of samples. To deal with the shortcomings of the traditional linear principal angles, nonlinear methods based on kernel trick were given [2].

A nonlinear transformation $T$ maps the samples $x_{k}(k=1, \mathrm{~L} m), y_{k}(k=1, \mathrm{~L} n)$ into a high dimensional feature space: $x_{k}{ }^{T} \varphi\left(x_{k}\right)$ and $y_{k}{ }^{T} \varphi\left(y_{k}\right)$. So the samples and the spanned spaces are written as:

\footnotetext{
${ }^{1}$ Corresponding author: Haikuan LIU, email: $\underline{\text { lzh_box@163.com }}$
} 


$$
\begin{aligned}
& \mathrm{U}_{\varphi(\mathrm{A})}=\operatorname{span}\left\{\varphi\left(x_{1}\right) ; \mathrm{L} ; \varphi\left(x_{\mathrm{m}}\right)\right\}=\left\{a: a=\sum_{i=1}^{m} \alpha_{i} \varphi\left(x_{i}\right), \alpha_{i} \in \mathrm{R}\right\}, \\
& \mathrm{U}_{\varphi(\mathrm{B})}=\operatorname{span}\left\{\varphi\left(y_{1}\right) ; \mathrm{L} ; \varphi\left(y_{\mathrm{n}}\right)\right\}=\left\{b: b=\sum_{i=1}^{n} \beta_{i} \varphi\left(y_{i}\right), \beta_{i} \in \mathrm{R}\right\} .
\end{aligned}
$$

Accordingly, the optimization problem (1) is rewritten as:

$$
\cos (\theta)=\max _{\varphi(\mathrm{u}) \in \mathrm{U}_{\varphi(\mathrm{A})}, \varphi(\mathrm{v}) \in \mathrm{U}_{\varphi(\mathrm{B})}} \varphi(\mathrm{u})^{\mathrm{T}} \varphi(\mathrm{v})
$$

subject to

$$
\varphi(\mathrm{u})^{\mathrm{T}} \varphi(\mathrm{u})=\varphi(\mathrm{v})^{\mathrm{T}} \varphi(\mathrm{u})=1 .
$$

From equation (3) and (4), we know that the explicit expression of the nonlinear transformation $T$ is unnecessary. A kernel function $k\left(x_{i}, x_{j}\right)$ satisfying the inner product $k\left(x_{i}, y_{j}\right)=\varphi\left(x_{i}\right)^{T} \varphi\left(x_{j}\right)$ is enough for the optimization problem (4) [3, 4]. Such kernel trick is widely used in machine learning, and several such kernel functions are widely discussed. The well-known inner product is the radial basis function $\varphi\left(x_{i}\right)^{T} \varphi\left(x_{j}\right)=e^{-\left\|x_{i}-x_{j}\right\|^{2} / \sigma^{2}}$. It is regarded all-purpose.

\section{EEG Signals}

EEG signals are very complex[4,5,6]. They often contain eye movement signals and are contaminated by different kinds of noises. More seriously, the amplitude of noises is often tens or hundreds of times higher than the EEG signals. So digital filtering is necessary.

The frequency of useful EEG signals ranges from $0.5 \mathrm{~Hz}$ to about $120 \mathrm{~Hz}$. According to Shannon sampling theorem, in order to recover the EEG signals from noisy signal, the sampling frequency is at least $240 \mathrm{~Hz}$. Considering the EEG signals is the mixture of signals from $0.5 \mathrm{~Hz}$ to $120 \mathrm{~Hz}$, the sampling frequency is usually up to $1 \mathrm{KHz}$. During our experiments, the sampling frequency is set up to $1.2 \mathrm{KHz}$.

It is well known that the blink time is about 0.2-0.4 second. During this time, about 300 samples generated. Intentional eye movement in different direction spends almost 1 second and generates 1000 samples. The signals inspired by intentional eye movement in different direction lasting about 1 second are called typical eye movement signals. In fact they are electrooculography (EOG) signals. We use EOG signals to recognize eye movements because it is more powerful, discernible and easy to get. In fact, many people use different methods to classify eye movement signals $[7,8,9]$.

\section{Experiments}

Suppose we are trying to capture four typical eye movement signals of an experimental subject. The experimental subject can be in five conditions: keeping eyes close calmly, moving eyes up, moving eyes down, moving eyes left, moving eyes right. These five conditions are looked as five random processes and denoted as $\mathrm{X}_{\text {close }}, \mathrm{X}_{\mathrm{up}}, \mathrm{X}_{\text {down }}, \mathrm{X}_{\text {left }}, \mathrm{X}_{\text {right }}$. Experimental subjects carried out these eye movements and EEG signals were recorded. Each of the special eye movements (for example, moving eyes up) is called a session, which usually lasted about 1 second. The experimental subjects sat in a chair and move the eyes up, down, left, right, or keeping close. These experiments were carried out time and time again. The sampled data sets were denoted as $D_{\text {close }}, D_{\text {up }}, D_{\text {down }}, D_{\text {left }}$, and $D_{\text {right }}$. They are from the random processes $\mathrm{X}_{\text {close }}, \mathrm{X}_{\text {up }}, \mathrm{X}_{\text {down }}, \mathrm{X}_{\text {left }}, \mathrm{X}_{\text {right }}$.

When groups of typical data were sampled for each session, the preparation is over.

All that remains now is to capture and identify the typical eye movement by online calculating the cosines. A flow of data from the experimental subject is recorded and denote as $D_{\text {test }}$. So we can get the cosines of kernel principal angles between $D_{\text {test }}$ and data set $D_{\text {close }}, D_{\text {up }}, D_{\text {down }}, D_{\text {left }}, D_{\text {right }}$ one by one. By this way, we can find that the samples from the same random process are more similar when measured by principal angles. The cosines of principal angles within random processes $\mathrm{X}_{\text {close }}$ are demonstrated in fig.1. The other cosines of kernel principal angles between eye moving up and eye 
close, eye moving down and eye close, eye moving left and eye close, eye moving right and eye close were shown in fig. 2.

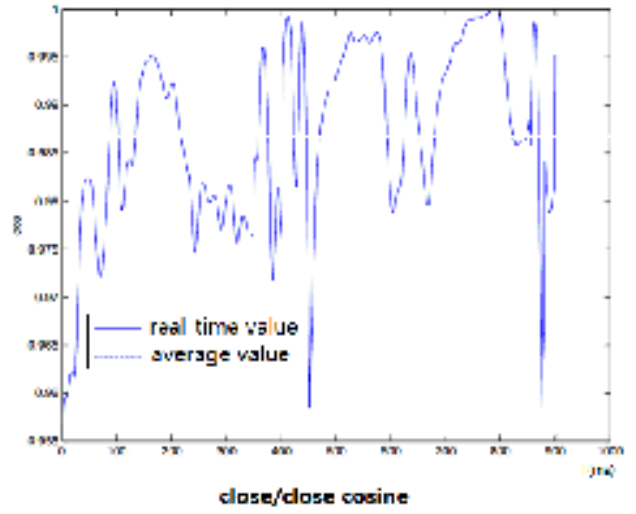

Fig. 1 Close and close similarity
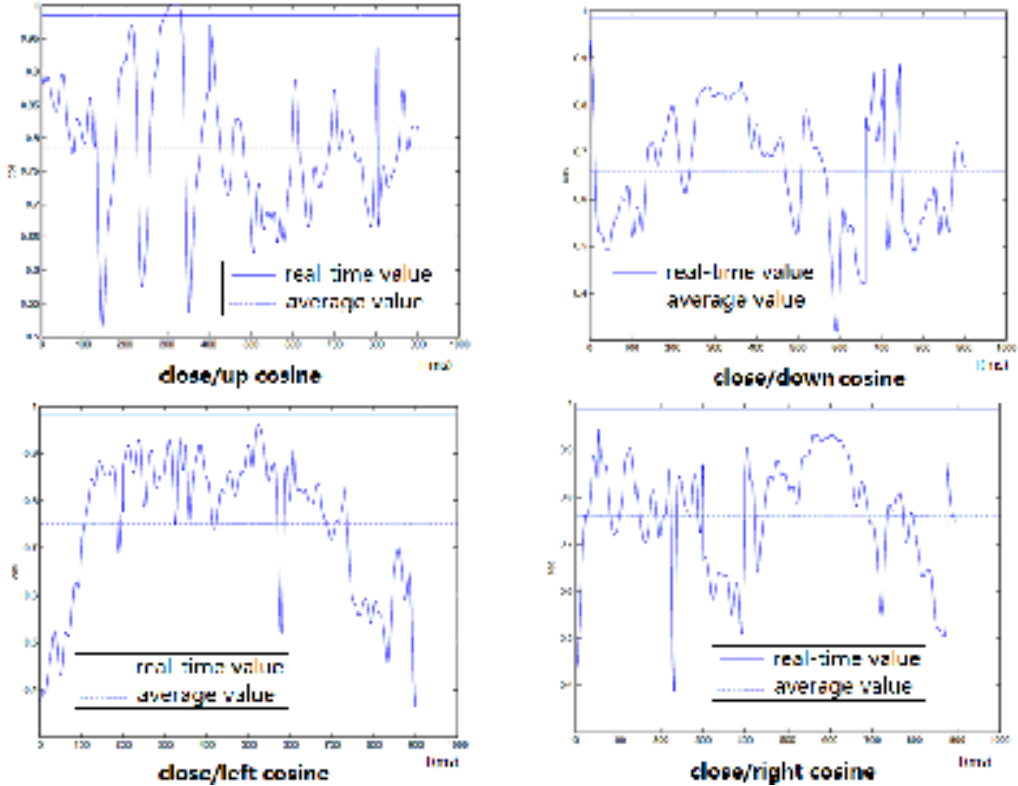

Fig. 2 Closed and other state similarity

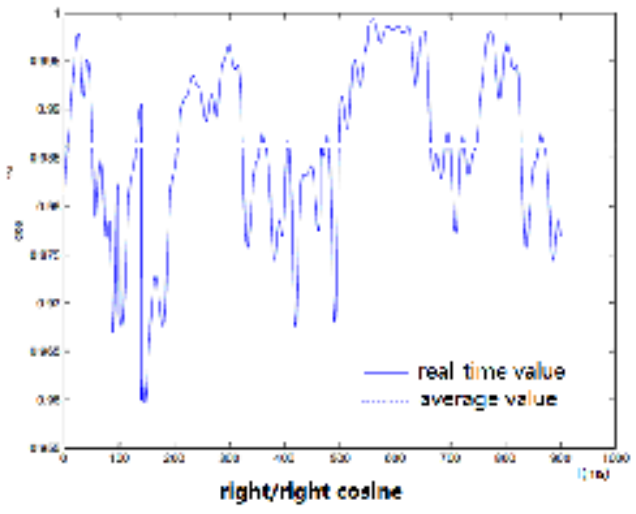

Fig. 3 Right and right similarity 

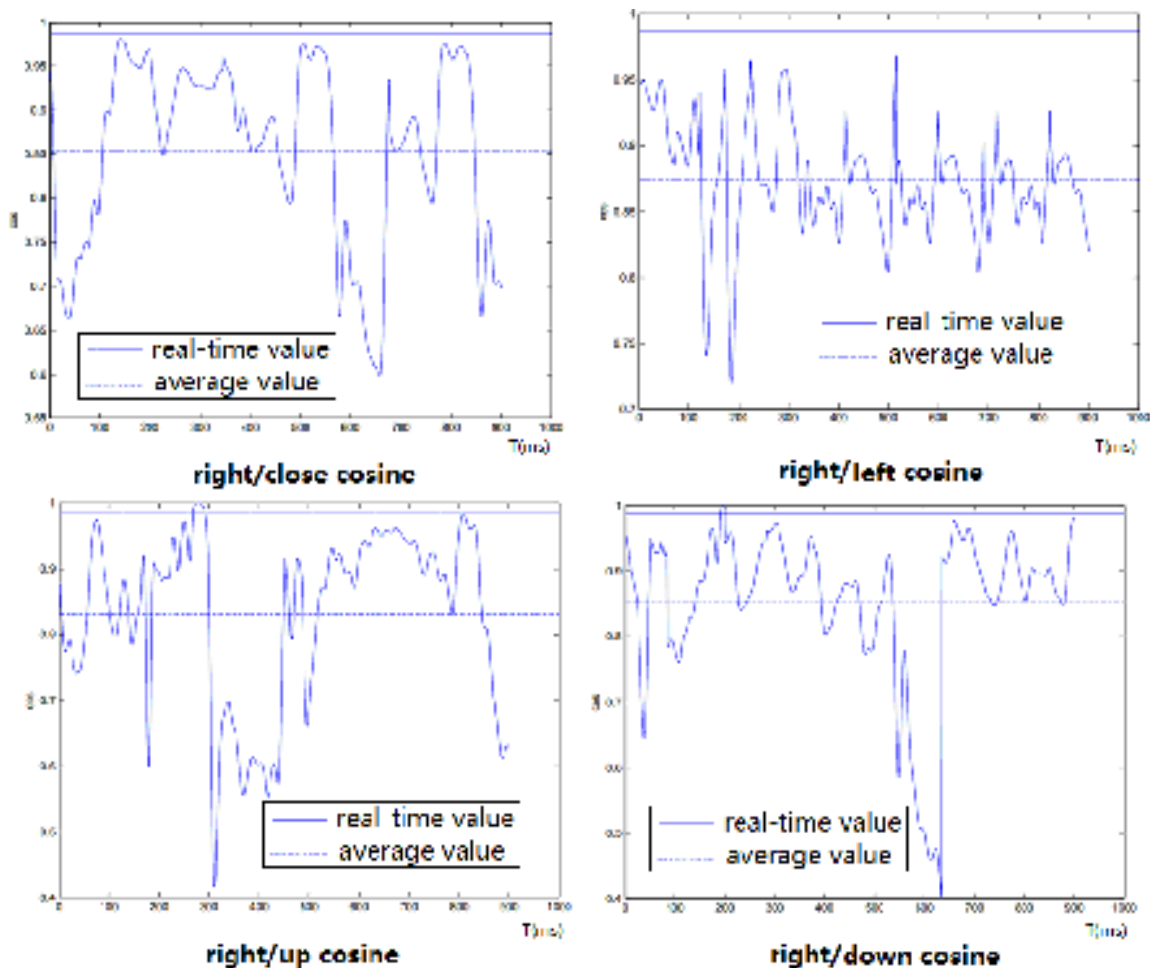

Fig. 4 Right and other state similarity

From fig. 1 to fig. 4 we can find that the cosines of principal angles within random processes $\left(\mathrm{X}_{\text {close }}\right.$ in fig. 1 and $X_{\text {right }}$ in fig. 3 ) approximate to 1 and the average is above 0.98 . The cosines across different random processes ( $\mathrm{X}_{\text {close }}$ and other processes in fig.2, $\mathrm{X}_{\text {right }}$ and other processes in fig.4) are much smaller and the averages are all below 0.90. By this way the special eye movement signals are captured and recognized.

\section{Conclusions}

We proposed a method to identify four typical eye movement signals hidden in EEGs. Eye movement signals contained in EEGs are often looked as artefacts to doctors. But they can be useful if the movements are launched for special purpose. Identifying typical eye movements can facilitate the communication between person and computer. Our experiment demonstrated the way to identify four typical eye movement signals by analyzing the EEG signals with kernel principal angles. The finally experiments show that suitable schedule and allocation can help with capturing special eye movement signals. With the help of kernel principal angles, we captured the eye movement signals hidden in EEGs and recognized them.

\section{References}

[1] H. Hotelling. Relations between two sets of variates. Biometrika, 28(1936): 321-372.

[2] V.N.Vapnik. The nature of statistical learning theory. 2000: Springer Verlag.

[3] L. Wolf, A. Shashua. Learning over sets using kernel principal angles. Journal of Machine Learning Research. 4 (2003) 913-931.

[4] L. Wolf, A. Shashua. Kernel principal angles for classification machines with applications to image sequence interpretation[C]// Computer Vision and Pattern Recognition. 2003 IEEE Computer Society Conference on. IEEE, 2003: 635-640.

[5] A. N. Belkacem, D. Shin, H. Kambara, N. Yoshimura, Y. Koike. Online classification algorithm for eye-movement-based communication systems using two temporal EEG sensors, Biomedical Signal Processing and Control. 16 (2015) 40-47. 
[6] T. Connie, O. M. Goh, B. J. Teoh. Multi-view gait recognition using a doubly-kernel approach on the Grassmann manifold[J]. Neurocomputing. 216(2016):534-542.

[7] S. Kawato, N. Tetsutani, Detection and tracking of eyes for gaze-camera control, Image Vis. Comput. 22 (2004) 1031-1038.

[8] Z.W. Zhu, Q. Ji, Robust real-time eye detection and tracking under variablelighting conditions and various face orientations, Comput. Vis. Image Underst.98(2005) 124-154.

[9] A. James, D. Vieira, B. Lo, A. Darzi, G.Z. Yang, Eye-gaze driven surgical work-flow segmentation, Med. Image Comput. Comput. Assist. Interv. 10 (2007)110-117. 